\title{
Upper Bounds for Ising Model Correlation Functions
}

\author{
COLIN J. THOMPSON * \\ The Institute for Advanced Study, Princeton, New Jersey USA
}

Received August 3, 1971

\begin{abstract}
A Griffiths correlation inequality for Ising ferromagnets is refined and is used to obtain improved upper bounds for critical temperatures. It is shown that, for non-negative external fields, the mean field magnetization is an upper bound for the magnetization of Ising ferromagnets.
\end{abstract}

\section{Introduction}

For each nonempty subset $R$ of an index set $\Lambda$ define

$$
\sigma_{R}=\prod_{i \in A} \sigma_{i}
$$

where $\sigma_{i}= \pm 1, i \in \Lambda$, is a set of Ising spins. In a given configuration of spins $\{\sigma\}=\left\{\sigma_{i}: i \in \Lambda\right\}$, the interaction energy is defined by

$$
E\{\sigma\}=-\sum_{R \subset A} J(R) \sigma_{R} .
$$

Thermodynamic averages of functions $f=f\{\sigma\}$ are defined by

$$
\langle f\rangle=\sum_{\{\sigma\}} f\{\sigma\} \exp (-\beta E\{\sigma\}) / \sum_{\{\sigma\}} \exp (-\beta E\{\sigma\})
$$

where sums extend over all configurations of spins. We denote

$$
\sigma_{R} \sigma_{S}=\sigma_{R S}
$$

where from the Definition (1.1) $R S$ is the set-theoretic symmetric difference $R \cup S-R \cap S$.

For ferromagnetic pair interactions, i.e., $J(R)$ non-negative and zero unless $R$ is a one or a two element subset of $\Lambda$ (one element subsets corresponding to interactions with an external field), Griffiths $[1,2,3]$ proved a number a correlation function inequalities which were subsequently generalized by Kelley and Sherman [4]. For the inter-

\footnotetext{
* On leave (1970-71) from Northwestern University, Evanston, Illinois 60201.
} Supported at IAS by a grant from the Alfred P. Sloan Foundation. 
action (1.2) with $J(R) \geqq 0$, these generalized Griffiths inequalities are

$$
\begin{array}{cc} 
& \left\langle\sigma_{R}\right\rangle \geqq 0, \\
\text { I. } & \frac{\partial}{\partial \beta J(S)}\left\langle\sigma_{R}\right\rangle=\left\langle\sigma_{R S}\right\rangle-\left\langle\sigma_{R}\right\rangle\left\langle\sigma_{S}\right\rangle \geqq 0,
\end{array}
$$

and for any $k \in R$

III.

$$
\left\langle\sigma_{R}\right\rangle \leqq \sum_{\substack{S \\ k \in S}} \tau(S)\left\langle\sigma_{R S}\right\rangle
$$

where

$$
\tau(S)=\tanh \beta J(S)
$$

and the sum in (1.7) extends over sets $S \in\{A \subset \Lambda, J(A)>0\}$.

It is to be noted that interactions with an external magnetic field $H>0$ can be included in the above by taking $J(R)=H$ for all one element subsets $R$ of $\Lambda$.

Ginibre [5] and Fortuin, Ginibre and Kasteleyn [6] have recently constructed a general framework in which inequalities of the type I and II are valid. Inequality II and its generalizations have been particularly useful in proving various existence theorems for phase transitions $[1,7]$ and for obtaining critical exponent inequalities [8]. The inequality III, which is the subject of this note, has been used primarily to obtain bounds for critical temperatures [3].

In the next section, we obtain a refinement of the inequality III and use it to obtain improved bounds for critical temperatures. In the final section, we show that, for non-negative external fields, the mean field magnetization is an upper bound for the magnetization of Ising ferromagnets with pair interactions.

\section{Refinement of the Inequality (1.7)}

In the following, we will make use of the identity

$$
\exp \left[\beta J(R) \sigma_{R}\right]=\cosh \beta J(R)\left[1+\tau(R) \sigma_{R}\right],
$$

where $\tau(R)$ is defined by (1.8). This result is easily proved by expanding the exponential and noting that $\left(\sigma_{R}\right)^{2}=1$. We will assume throughout that $J(R) \geqq 0$.

Writing

$$
\exp (-\beta E\{\sigma\})=\prod_{R \subset A} \exp \left[\beta J(R) \sigma_{R}\right]
$$


and applying the identity (2.1) to the term in (2.2) corresponding to the subset $S \subset \Lambda$, we obtain immediately from the definition (1.3) that

$$
\left\langle\sigma_{R}\right\rangle=\left[\left\langle\sigma_{R}\right\rangle_{S}+\tau(S)\left\langle\sigma_{R S}\right\rangle_{S}\right]\left[1+\tau(S)\left\langle\sigma_{S}\right\rangle_{S}\right]^{-1}
$$

where $\langle\ldots\rangle_{S}$ denotes an average for the system (1.2) with $J(S)=0$. Interchanging $R$ and $R S$ in (2.3) gives

$$
\left\langle\sigma_{R S}\right\rangle=\left[\left\langle\sigma_{R S}\right\rangle_{S}+\tau(S)\left\langle\sigma_{R}\right\rangle_{S}\right]\left[1+\tau(S)\left\langle\sigma_{S}\right\rangle_{S}\right]^{-1} .
$$

Combining (2.3) and (2.4) then gives

$$
\begin{aligned}
\left\langle\sigma_{R}\right\rangle & =\tau(S)\left\langle\sigma_{R S}\right\rangle+\left[\left(1-\tau(S)^{2}\right)\left\langle\sigma_{R}\right\rangle_{S}\right]\left[1+\tau(S)\left\langle\sigma_{S}\right\rangle_{S}\right]^{-1} \\
& \leqq \tau(S)\left\langle\sigma_{R S}\right\rangle+\left(1-\tau(S)^{2}\right)\left\langle\sigma_{R}\right\rangle_{S}
\end{aligned}
$$

where use has been made of (1.5), i.e., $\left\langle\sigma_{S}\right\rangle_{S} \geqq 0$.

Let now $S_{1}, S_{2}, \ldots, S_{n}$ be any family of subsets of $\Lambda$. By repeated iteration of (2.5), we obtain

$$
\begin{aligned}
\left\langle\sigma_{R}\right\rangle \leqq \tau\left(S_{1}\right) & \left\langle\sigma_{R S_{1}}\right\rangle+\left(1-\tau\left(S_{1}\right)^{2}\right) \tau\left(S_{2}\right)\left\langle\sigma_{R S_{2}}\right\rangle_{S_{2}} \\
& +\cdots+\left(1-\tau\left(S_{1}\right)^{2}\right) \ldots\left(1-\tau\left(S_{n}\right)^{2}\right)\left\langle\sigma_{R}\right\rangle_{S_{1}, S_{2}, \ldots S_{n}},
\end{aligned}
$$

i.e.

$$
\begin{aligned}
\left\langle\sigma_{R}\right\rangle \leqq & \sum_{j=1}^{n} \tau\left(S_{j}\right)\left\{\prod_{i=0}^{j-1}\left(1-\tau\left(S_{i}\right)^{2}\right)\right\}\left\langle\sigma_{R S_{j}}\right\rangle_{S_{1}, \ldots, S_{j-1}} \\
& +\prod_{i=1}^{n}\left(1-\tau\left(S_{i}\right)^{2}\right)\left\langle\sigma_{R}\right\rangle_{S_{1}, \ldots, S_{n}}
\end{aligned}
$$

where $\tau\left(S_{0}\right) \equiv 0$.

It is to be noted that if the family $\left\{S_{i}\right\}=\mathscr{A}$, the set of subsets of $\Lambda$ excluding $R$ such that $S_{i} \cap R \neq \phi$ and $J\left(S_{i}\right)>0, i=1,2, \ldots n$,

$$
\left\langle\sigma_{R}\right\rangle_{S_{1}, \ldots, S_{n}}=\tau(R) \text {. }
$$

Also, because of the monotonicity property (1.6)

$$
\left\langle\sigma_{R S_{j}}\right\rangle_{S_{1}, \ldots, S_{j-1}} \leqq\left\langle\sigma_{R S_{j}}\right\rangle \text {. }
$$

It follows that if $\left\{S_{i}, i=1,2, \ldots, n\right\}=\mathscr{A}$ and $S_{n+1}=R,(2.6),(2.7)$, and (2.8) give

$$
\left\langle\sigma_{R}\right\rangle \leqq \sum_{j=1}^{n+1} \tau\left(S_{j}\right)\left\{\prod_{i=0}^{j-1}\left(1-\tau\left(S_{i}\right)^{2}\right)\right\}\left\langle\sigma_{R S_{j}}\right\rangle
$$

where use has been made of $\left\langle\sigma_{R R}\right\rangle=\left\langle\sigma_{R}^{2}\right\rangle=1$. Obviously, the best inequality from (2.9) is obtained by choosing an ordering for $S_{1}, S_{2}, \ldots, S_{n}$ which minimizes the right hand side.

For a set of $N$ pair-wise interacting spins in the presence of an external magnetic field $H$, we choose $R=\{r\}(J(R)=H), S_{j}=\left\{r, s_{j}\right\}$ 
$j=1,2, \ldots, N-1, S_{N}=R$, such that $s_{i} \neq s_{j}, i \neq j$, and $s_{j} \neq r$. From (2.9) (with $n=N-1$ ), we then obtain

$$
\begin{aligned}
\left\langle\sigma_{r}\right\rangle \leqq & \prod_{j=1}^{N-1}\left(1-\tau\left(r, s_{j}\right)^{2}\right) \tanh \beta H \\
& +\sum_{j=1}^{N-1} \tau\left(r, s_{j}\right)\left\{\prod_{i=0}^{j-1}\left(1-\tau\left(r, s_{i}\right)^{2}\right)\right\}\left\langle\sigma_{s_{j}}\right\rangle,
\end{aligned}
$$

where $\tau(r, s)=\tanh \beta J_{r s}, J_{r s}$ is the coupling constant between spins $r$ and $s$, and $\tau\left(r, s_{0}\right) \equiv 0$.

For a translationally invariant system $\left\langle\sigma_{k}\right\rangle=m_{N}(H, \beta)$ is the magnetization per spin for all $k$. It follows from (2.10) that if

$$
G(\beta)=\sum_{j=1}^{N-1} \tau\left(r, s_{j}\right)\left\{\prod_{i=0}^{j-1}\left(1-\tau\left(r, s_{i}\right)^{2}\right)\right\}<1
$$

the spontaneous magnetization $m_{0}(\beta)=\lim _{H \rightarrow 0+} \lim _{N \rightarrow \infty} m_{N}(H, \beta)$ vanishes, and hence that a solution of

$$
G\left(\beta_{0}\right)=1, \quad \beta_{0}=\left(k T_{0}\right)^{-1}
$$

gives an upper bound $T_{0}$ for the critical temperature $T_{c}$.

For example, if there are nearest neighbor interactions only on a lattice with coordination number $q$,

$$
G(\beta)=\tanh (\beta J) \sum_{j=0}^{q-1}\left(1-\tanh ^{2} \beta J\right)^{j}
$$

where $J$ is the coupling constant between nearest neighbor spins. For the square lattice $(q=4),(2.12)$ and $(2.13)$ give $\tanh \left(\beta_{0} J\right)=0.29 \ldots$, which is to be compared with the mean field value 0.25 [3], Fisher's [9] selfavoiding walk bound $0.37 \ldots$, and the exact value $\sqrt{2}-1=0.414 \ldots$. The bounds obtained from (2.12) and (2.13) of course improve with increasing coordination number.

\section{Mean Field Bound for the Magnetization}

For a set of $N$ Ising spins with ferromagnetic pair interactions only in the presence of an external magnetic field $H \geqq 0$, the choice $R=\{r\}$, $S=\{r, s\}$ in (2.3) gives

$$
\left\langle\sigma_{r}\right\rangle=\left[\left\langle\sigma_{r}\right\rangle_{S}+\tanh \left(\beta J_{r s}\right)\left\langle\sigma_{s}\right\rangle_{S}\right]\left[1+\tanh \left(\beta J_{r s}\right)\left\langle\sigma_{r} \sigma_{s}\right\rangle_{S}\right]^{-1}
$$

where $J_{r s} \geqq 0$ is the coupling constant between spins $r$ and $s$. From the monotonicity property (1.6), $\left\langle\sigma_{r} \sigma_{s}\right\rangle_{S} \geqq\left\langle\sigma_{r}\right\rangle_{S}\left\langle\sigma_{s}\right\rangle_{S}$. Also, since 
$0 \leqq\left\langle\sigma_{s}\right\rangle_{S} \leqq 1,\left\langle\sigma_{s}\right\rangle_{S} \tanh \beta J_{r_{s}} \leqq \tanh \left(\beta J_{r s}\left\langle\sigma_{s}\right\rangle_{S}\right)$. Using these results in (3.1), we obtain

$$
\left\langle\sigma_{r}\right\rangle \leqq \tanh \left(\beta J_{r s}\left\langle\sigma_{s}\right\rangle_{S}+g\left(\left\langle\sigma_{r}\right\rangle_{S}\right)\right)
$$

where $g(z)=\tanh ^{-1} z$. Hence, since $\left\langle\sigma_{s}\right\rangle_{S} \leqq\left\langle\sigma_{s}\right\rangle$,

$$
g\left(\left\langle\sigma_{r}\right\rangle\right) \leqq \beta J_{r s}\left\langle\sigma_{s}\right\rangle+g\left(\left\langle\sigma_{r}\right\rangle_{S}\right)
$$

Iterating (3.3) until all bonds $J_{r, s}>0$ have been eliminated, we then obtain, using (2.7)

i.e.

$$
g\left(\left\langle\sigma_{r}\right\rangle\right) \leqq \sum_{s \neq r} \beta J_{r s}\left\langle\sigma_{s}\right\rangle+\beta H
$$

$$
\left\langle\sigma_{r}\right\rangle \leqq \tanh \left(\sum_{s \neq r} \beta J_{r s}\left\langle\sigma_{s}\right\rangle+\beta H\right)
$$

For a translationally invariant system $\left\langle\sigma_{r}\right\rangle=m_{N}(H, \beta)$ is the magnetization per spin for all $r$. Taking the limit $N \rightarrow \infty$ in (3.4), we then obtain

$$
0 \leqq m \leqq \tanh (\beta \alpha m+\beta H), \quad \text { for } \quad H \geqq 0,
$$

where $m=\lim _{N \rightarrow \infty} m_{N}(H, \beta)$, and from translational invariance,

$$
\alpha=\sum_{s \neq r} J_{r s}
$$

is independent of $r$.

The positive solution of

$$
m^{*}=\tanh \left(\beta \alpha m^{*}+\beta H\right), \quad H \geqq 0
$$

is the mean field magnetization. From (3.5) we then obtain

$$
0 \leqq m \leqq m^{*}, \quad \text { for } \quad H \geqq 0 .
$$

Notice also, from (3.8), that the mean field critical temperature $T^{*}$ given from (3.7) by $\beta^{*}=\left(k T^{*}\right)^{-1}=\alpha^{-1}$ is necessarily an upper bound for the true critical temperature.

Acknowledgements. I am grateful to J. Ginibre for remarks which led to the general form of the inequality (2.9) and to H. Falk for suggesting that the inequality (3.8) was probably true. I would like also to thank Dr. Carl Kaysen for his kind hospitality at The Institute for Advanced Study.

\section{References}

1. Griffiths, R.B.: J. Math. Phys. 8, 478 (1967).

2. - J. Math. Phys. 8, 484 (1967).

3. - Commun. math. Phys. 6, 121 (1967).

5 Commun. math. Phys, Vol. 24 
4. Kelly, D. G., Sherman, S.: J. Math. Phys. 9, 466 (1968).

5. Ginibre, J.: Commun. math. Phys. 16, 310 (1970).

6. Fortuin, C.M., Ginibre,J., Kasteleyn,P.W.: Correlation inequalities on some partially ordered sets. Preprint, January 1971.

7. Dyson, F. J.: Commun. math. Phys. 12, 91, 212 (1969).

8. Buckingham, M. J., Gunton, J. D.: Phys. Rev. 178, 848 (1969); Fisher, M.E.: Phys. Rev. 180, 594 (1969).

9. Fisher, M.E.: Phys. Rev. 162, 480 (1967).

C. J. Thompson

The Institute for Advanced Study

Princeton, N. J. 08540, USA 